\title{
Magnetization depth profile of (Fe/Dy) multilayers
}

\author{
Alexandre Tamion ${ }^{\mathrm{a}, \mathrm{b}, *}$, Frédéric Ott ${ }^{\mathrm{c}}$, Pierre-Emmanuel Berche ${ }^{\mathrm{a}}$, Etienne Talbot ${ }^{\mathrm{a}}$, Catherine Bordel $^{\mathrm{a}}$, \\ Didier Blavette ${ }^{\mathrm{a}}$ \\ a Groupe de Physique des Matériaux UMR CNRS 6634,Université de Rouen, Avenue de l'Université, BP12, F-76801 Saint Etienne du Rouvray Cedex, France

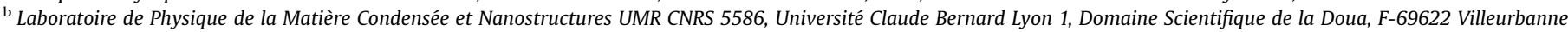 \\ Cedex, France \\ ${ }^{\mathrm{C}}$ Laboratoire Léon Brillouin, CEA/CNRS UMR12, Centre d'Etudes de Saclay, 91191 Gif sur Yvette Cedex, France
}

\begin{abstract}
A B S T R A C T
An experimental and numerical study of the magnetization in (Fe $3 \mathrm{~nm} / \mathrm{Dy} 2 \mathrm{~nm}$ ) multilayers is presented. The samples were thermally evaporated under ultra-high vacuum at two different substrate temperatures, 320 and $570 \mathrm{~K}$. In order to get the magnetization depth profile of these transition metal/ rare earth (TM/RE) multilayers, a fine investigation of the structural, chemical, and magnetic properties was carried out. The samples were studied by X-ray reflectivity (XRR), high resolution transmission electron microscopy (HRTEM), conversion electron Mössbauer spectrometry (CEMS), SQUID magnetometry and polarized neutron reflectivity (PNR). Magnetization profiles were obtained by Monte Carlo simulations to support the PNR fits. The key role of the crystalline structure is emphasized by magnetic depth profile measurements performed using polarized neutron reflectometry. The antiparallel configuration of Fe and Dy layers' magnetizations was evidenced, as well as the perpendicular magnetic anisotropy (PMA), especially in the case of the sample prepared at $570 \mathrm{~K}$.
\end{abstract}

\section{Introduction}

Nanostructured artificial materials are of great interest since the two-dimensional character of these systems is responsible for exciting phenomena such as giant magnetoresistance or perpendicular magnetic anisotropy. The perpendicular magnetic anisotropy (PMA) in (TM/RE) multilayers has been studied very intensively for several decades [1-17], but its origin is still controversial. Since Tb has the largest local anisotropy constant, most of the studies have been devoted to (Fe/Tb) multilayers. However, (Fe/Dy) multilayers exhibit similar properties and have been less studied $[2,4,10,14,18]$. A recent study [19] has shown that the PMA observed in amorphous (Fe/Dy) multilayers mainly results from the interfacial Fe-Dy amorphous alloy formed by chemical mixing rather than from the composition modulation [1] or from the symmetry breaking at the interfaces [8].
The aim of this work is to understand the influence of the deposition temperature on the magnetic properties, especially the magnetization depth profile of (Fe/Dy) multilayers. A careful determination of the structural and chemical features is essential to understand the macroscopic magnetic properties of these multilayers. (Fe $3 \mathrm{~nm} /$ Dy $2 \mathrm{~nm}$ ) multilayers were deposited on $\mathrm{Si}$ substrates. Structural, chemical and magnetic characterizations were carried out. Monte Carlo simulations were performed to complement and validate the results obtained by polarized neutron reflectivity (PNR).

\section{Experiment}

\subsection{Multilayer deposition conditions}

(Fe $3 \mathrm{~nm} /$ Dy $2 \mathrm{~nm}$ ) multilayers were deposited by thermal evaporation under ultra-high vacuum on rotating ( 001$)$-oriented $\mathrm{Si}$ wafers. The deposition rate was about $0.01 \mathrm{~nm} / \mathrm{s}$ and the thicknesses were controlled by a quartz monitor. The background pressure was $10^{-8} \mathrm{~Pa}$. The films were covered with a $5 \mathrm{~nm} \mathrm{Cr}$ top layer to prevent oxidation. The substrate temperature $\left(T_{\mathrm{S}}\right)$ was 
successively fixed at 320 and $570 \mathrm{~K}$. A typical specimen consists of 30 bilayers for conversion electron Mössbauer spectrometry (CEMS), SQUID magnetometry and high resolution transmission electron microscopy (HRTEM). For X-ray reflectivity (XRR) and PNR, samples made of 10 bilayers were prepared to make sure that the thickness and roughness were the same for all bilayers.

\subsection{Cross-sectional TEM specimen preparation}

The structure of the layers was investigated by transmission electron microscopy (TEM) experiments using a Tecnaï G2 fieldemission microscope working at $200 \mathrm{kV}$. The samples for TEM studies were thinned by mechanical grinding and ion-milling (using a precision ion polishing system) to achieve electron transparency. TEM analyses were performed both on crosssectional and plane-view specimens.

\section{Results and discussion}

\subsection{Chemical and structural characterizations}

\subsubsection{X-ray reflectivity (XRR)}

Prior to the PNR experiments, the samples were characterized by XRR, in order to know the periodicity of the multilayers. XRR was performed with a Co anode of $0.179 \mathrm{~nm}$ wavelength on a Bruker D8-advance diffractometer. Fig. 1 shows the XRR curves of the multilayers deposited at 320 and $570 \mathrm{~K}$. The curves were adjusted with the SimulReflec Software [20]. The small intensity of the Bragg peaks comes from the low optical contrast between Fe and Dy (2\%).

The interest of these measurements lies in the evolution of the Bragg peaks. Since the first one is convoluted with the Kiessig fringes, our attention will be focused on the second Bragg peak (located with an arrow on the curves). Comparing the peak position for the multilayers deposited at 320 and $570 \mathrm{~K}$, we can notice a peak shift toward larger $\theta$, resulting from a $4 \%$ reduction of the period between 320 and $570 \mathrm{~K}$. This is attributed to a change in the quartz balance calibration at higher temperatures. It can be noted that the peak intensity decreases with the increase in the substrate temperature. This behavior can result either from the chemical mixing between Fe and Dy at the interfaces, or from the increase of the layers' roughness. According to the tomographic atom probe (TAP) results [19], the decrease of the Bragg peak intensity is due to the mixing of Fe and Dy atoms at the interfaces. As a conclusion concerning the chemical features of the

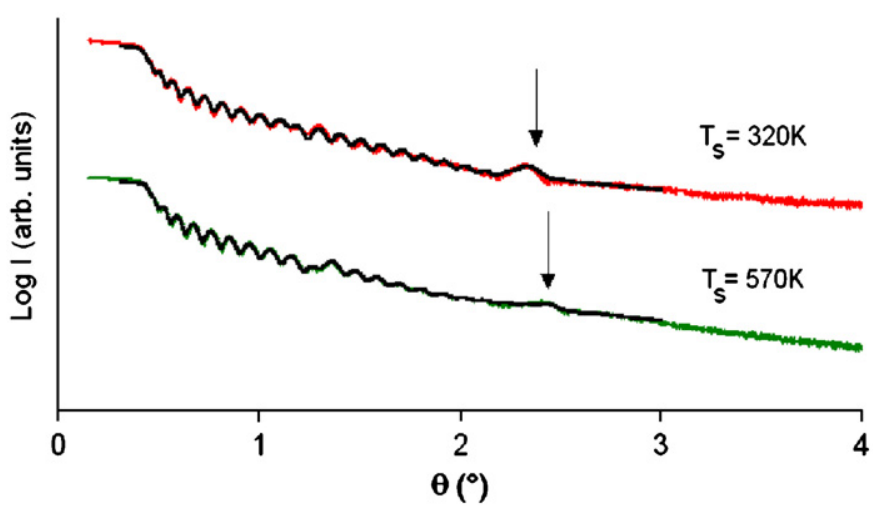

Fig. 1. Experimental X-ray reflectivity curves of (Fe $3 \mathrm{~nm} / \mathrm{Dy} 2 \mathrm{~nm}$ ) multilayers deposited at 320 and $570 \mathrm{~K}$. The fits to the data are presented in black. The arrows indicate the position of the second Bragg peak. stacks, it can be stated that the increase in the substrate temperature from 320 to $570 \mathrm{~K}$ leads to a chemical mixing between adjacent layers. In order to probe the structural order of these samples, X-ray diffraction experiments were performed. The diffractograms were identical for the two substrate temperatures, exhibiting only a very intense peak coming from the monocrystalline $\mathrm{Si}$ substrate. Although it is difficult to draw up a conclusion from such a weak evidence, it can be suggested that there is no long range order in these samples. Other investigations enabled the structure to be characterized.

\subsubsection{Mö̈sbauer spectrometry}

Conversion electron ${ }^{57} \mathrm{Fe}$ Mos̈sbauer spectrometry was used to obtain structural information concerning the Fe atoms of the multilayers. The samples were characterized in zero-applied field at room temperature, under normal incidence, with a home-made helium-methane gas proportional counter, with ${ }^{57} \mathrm{Co}$ in an $\mathrm{Rh}$ matrix as the source. The experimental spectra were fitted with a least-square technique using the histogram method. The Mos̈sbauer spectra at $300 \mathrm{~K}$ of (Fe $3 \mathrm{~nm} / \mathrm{Dy} 2 \mathrm{~nm}$ ) multilayers deposited at 320 and $570 \mathrm{~K}$ are shown in Fig. 2(a) and (b). For the sample prepared at $320 \mathrm{~K}$, the Mos̈sbauer spectrum exhibits a small sextet characteristic of the crystalline $\alpha$-Fe structure and an intense central peak that is attributed to amorphous Fe [5]. For $T_{\mathrm{S}}=570 \mathrm{~K}$, the spectrum is characteristic of a disordered alloyed structure, as already observed on other amorphous Fe-RE alloys [21,22]. This result is in agreement with the TAP analysis [19] and the XRR curve (Fig. 1) showing a penetration of the Dy layers by Fe atoms for a preparation temperature of $570 \mathrm{~K}$.

The multilayers are therefore structurally mainly disordered, but $\alpha$-Fe nanocrystals do exist, at least for $T_{\mathrm{S}}=320 \mathrm{~K}$, according to these CEMS analyses.

\subsubsection{HRTEM observations}

The layered structure of the samples was observed directly by means of HRTEM. Fig. 3 shows HRTEM and TEM images of (Fe $3 \mathrm{~nm} /$ Dy $2 \mathrm{~nm}$ ) multilayers prepared at 320 and $570 \mathrm{~K}$. Similar images were obtained for both of them. It can be observed on the low magnification image (Fig. 3(b)) that these samples have a regular structure. The dark layers correspond to the Fe layers and the bright layers to the Dy layers. It is important to note the presence of dark spots in the Fe layers, preferentially located close to the $\mathrm{Si}$ substrate. These spots are attributed to a diffraction contrast coming from nanocrystalline clusters in the Fe layers. On the high resolution image (Fig. 3(a)), we can see, indeed, that the first-deposited Fe layers are polycrystalline, whereas no structural
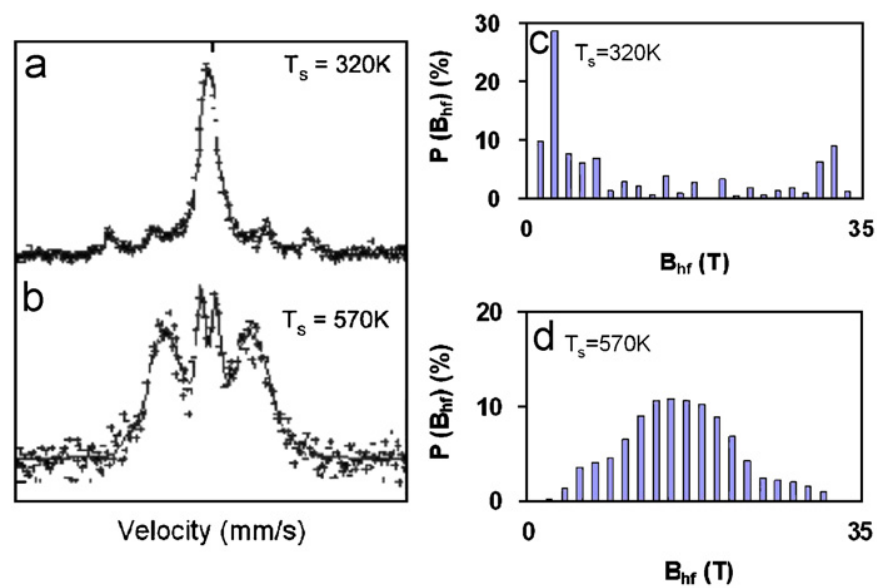

Fig. 2. Mössbauer spectra (a), (b) and hyperfine field $\left(B_{\mathrm{hf}}\right)$ distributions (c), (d) at $300 \mathrm{~K}$ for (Fe $3 \mathrm{~nm} / \mathrm{Dy} 2 \mathrm{~nm}$ ) multilayers deposited at $320 \mathrm{~K}$ (a, c) and $570 \mathrm{~K}$ (b, d). 

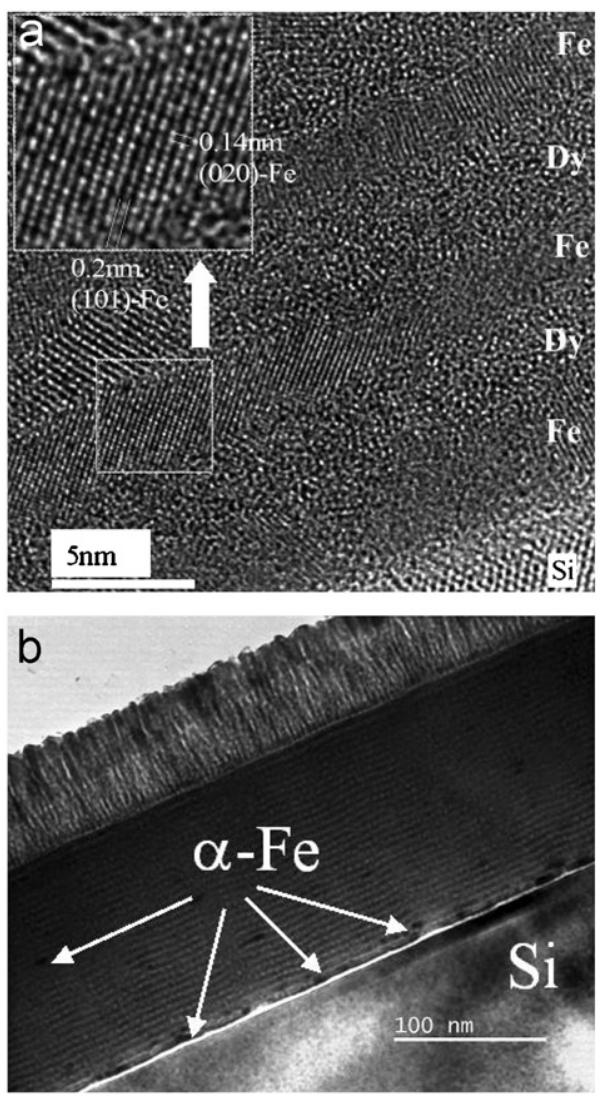

Fig. 3. HRTEM and TEM images of (Fe $3 \mathrm{~nm} / \mathrm{Dy} 2 \mathrm{~nm}$ ) multilayers deposited at $320 \mathrm{~K}(\mathrm{a})$ and $570 \mathrm{~K}(\mathrm{~b})$

order is visualized in the Dy layers. The crystalline Fe clusters previously detected by CEMS were thus found to be mainly positioned near the $\mathrm{Si}$ substrate. Fig. 4 shows the electron diffraction patterns of the same multilayers. Broad diffraction rings are observed on Fig. 4(a) and (b), indicating that the Dy and Fe layers are rather amorphous. However, the ring diameters of the multilayer prepared at $320 \mathrm{~K}$ (Fig. 4(a)) are characteristic of the d-spacings of $\alpha$-Fe. The diffraction pattern for $T_{\mathrm{S}}=570 \mathrm{~K}$ (Fig. 4(b)) exhibits three broad diffraction rings corresponding again to the crystal d-spacings of the $\alpha$-Fe phase $(0.20,0.14$ and $0.11 \mathrm{~nm})$ along the (110), (020) and (211) directions, respectively. The decrease in the intensity and number of rings reflects a lowering of the $\alpha$-Fe fraction in the samples with the rise of the substrate temperature from 320 to $570 \mathrm{~K}$. This decrease might be due to a greater chemical mixing between the layers. Indeed, the chemical mixing increases [19] when the substrate temperature is raised.

Thus, the fraction of pure Fe in the Fe layers decreases, which leads to a reduction in the $\alpha$-Fe fraction in the sample, in agreement with the CEMS spectra (Fig. 2(a), (b)).

\subsection{Magnetic characterizations}

\subsubsection{Mössbauer spectrometry}

The hyperfine field distributions are presented in Fig. 2(c) and (d). The low-field contributions $\left(B_{\mathrm{hf}}<5 \mathrm{~T}\right.$ ) observed for $T_{\mathrm{S}}=320 \mathrm{~K}$ (c) are characteristic of paramagnetic Fe, whereas the high-field component $\left(B_{\mathrm{hf}}=33 \mathrm{~T}\right)$ is the signature of ferromagnetic $\alpha$-Fe [5]. In the case of $T_{\mathrm{S}}=570 \mathrm{~K}(\mathrm{~d})$, the wide distribution of hyperfine field, associated with a ferromagnetic disordered phase, indicates that the Fe magnetic moment is highly dependent on its local environment [21,22]. Moreover, the fit to the data leads to a Mössbauer angle, i.e. the mean angle between the Fe magnetic
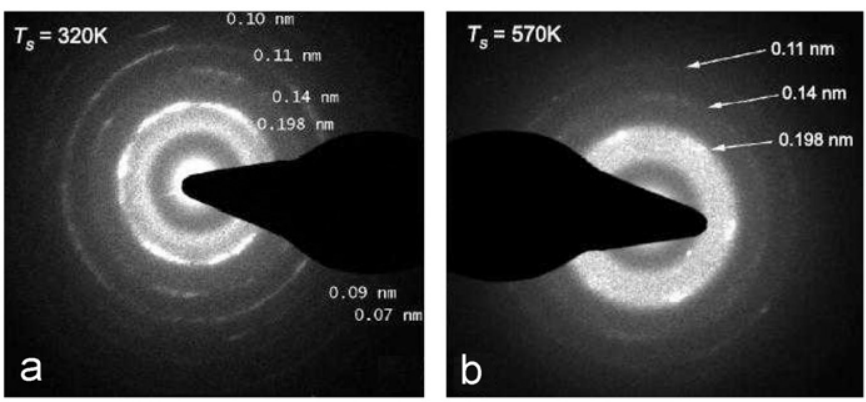

Fig. 4. Electron diffraction patterns obtained on (Fe $3 \mathrm{~nm} /$ Dy $2 \mathrm{~nm}$ ) multilayers deposited at $320 \mathrm{~K}$ (a) and $570 \mathrm{~K}$ (b).

moments and the normal to the film plane, of $7^{\circ}$ for the multilayer deposited at $570 \mathrm{~K}$. The magnetic anisotropy is therefore almost perpendicular to the substrate.

\subsubsection{SQUID magnetometry}

Magnetic hysteresis loops of (Fe $3 \mathrm{~nm} / \mathrm{Dy} 2 \mathrm{~nm}$ ) multilayers were obtained with a quantum design MPMS-XL SQUID. Fig. 5 shows the hysteresis loops measured at 300 and $100 \mathrm{~K}$ on samples prepared at 320 and $570 \mathrm{~K}$. The magnetic field was successively applied in the sample plane and along the normal to the film plane. It can be observed in Fig. 5(a) that the sample prepared at $320 \mathrm{~K}$ displays a ferromagnetic order which is quasi-isotropic at $300 \mathrm{~K}$. This signal is attributed to the $\alpha$-Fe nanocrystalline clusters detected by CEMS and TEM, the rest of the sample being nonmagnetic at $300 \mathrm{~K}$. The sample prepared at $570 \mathrm{~K}$ exhibits PMA at $300 \mathrm{~K}$ (Fig. 5(b)), in agreement with the Mössbauer angle $\left(7^{\circ}\right)$ previously obtained. We can, however, notice that the hysteresis loops obtained for $T_{\mathrm{S}}=570 \mathrm{~K}$ at $300 \mathrm{~K}$ reveal the existence of two magnetic phases in the sample, since an easy-plane signal, which is evidenced by the existence of a remanent magnetization, is detected in the "parallel" direction. This contribution to the total signal is likely to be due to the first bilayers of the sample, which have been found to contain $\alpha$-Fe, according to the TEM observations (Fig. 3). At $100 \mathrm{~K}$, Fig. 5(c) shows that the magnetic anisotropy of the sample prepared at $320 \mathrm{~K}$ is neither planar nor perpendicular to the film plane, which could be due to an easycone anisotropy [23]. In contrast, a strong uniaxial perpendicular magnetic anisotropy is clearly evidenced in Fig. 5(d) for the multilayer deposited at $570 \mathrm{~K}$ and measured at $100 \mathrm{~K}$.

\subsection{Polarized neutron reflectivity}

(Fe $3 \mathrm{~nm} /$ Dy $2 \mathrm{~nm}$ ) multilayers deposited at 320 and $570 \mathrm{~K}$ have been investigated by polarized neutron reflectometry (PNR) in order to obtain their magnetization depth profile in the multilayers. We performed the experiments on (Fe $3 \mathrm{~nm} /$ Dy $2 \mathrm{~nm})_{10}$ multilayers on the PRISM reflectometer of the Laboratoire Léon Brillouin (CNRS-CEA Saclay), with polarized neutrons and polarization analysis. The non-spin-flip (NSF) reflectivities named $R^{++}$and $R^{--}$were measured. The ' + ' and '-' signs indicate whether the neutron spin direction is parallel or antiparallel to the applied field $\mathbf{H}$. The polarization of the beam was $97.5 \%$, providing very good quality '++' and '--' cross section measurements. The background noise (two counts per minute) was subtracted from the measured data, and the reflectivities were corrected for the varying illumination. It is important to note that no signal was observed when measuring the spin-flip channels $\left(R^{+-}\right.$and $\left.R^{-+}\right)$. In PNR measurements, the neutrons are sensitive to the in-plane magnetization component only. The neutron reflectivity was measured at 300 and $100 \mathrm{~K}$ in order to obtain the magnetization depth profile as a function of temperature. 


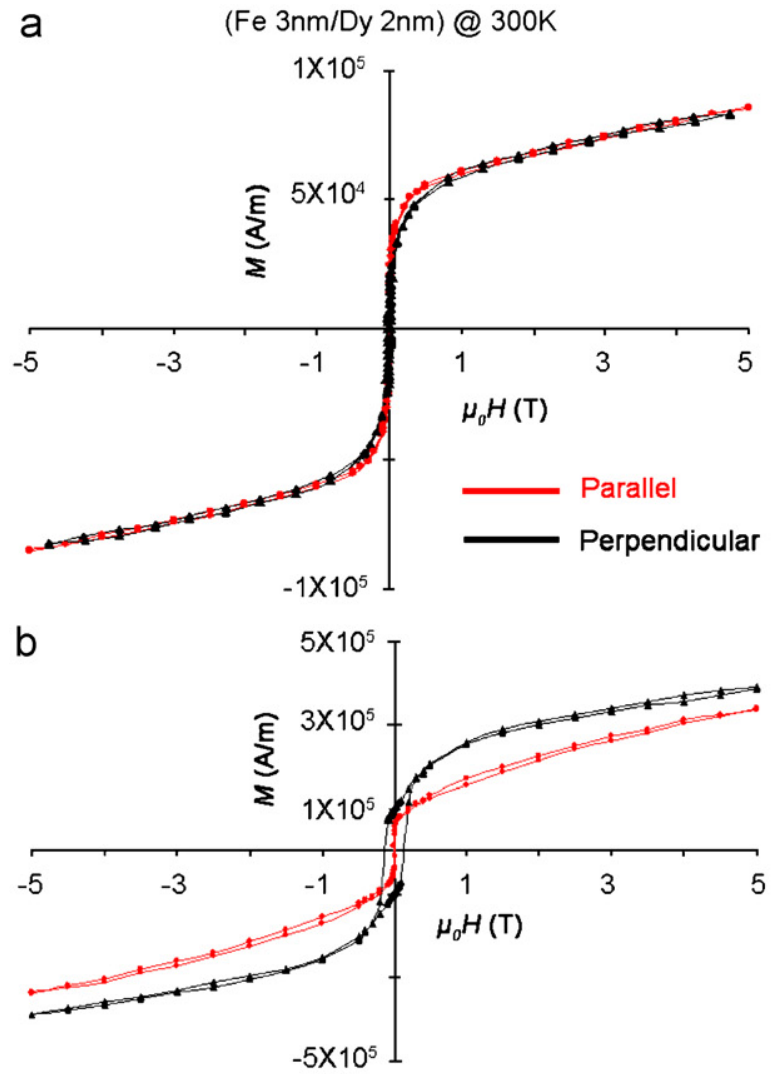

(Fe 3nm/Dy 2nm) @ 100K
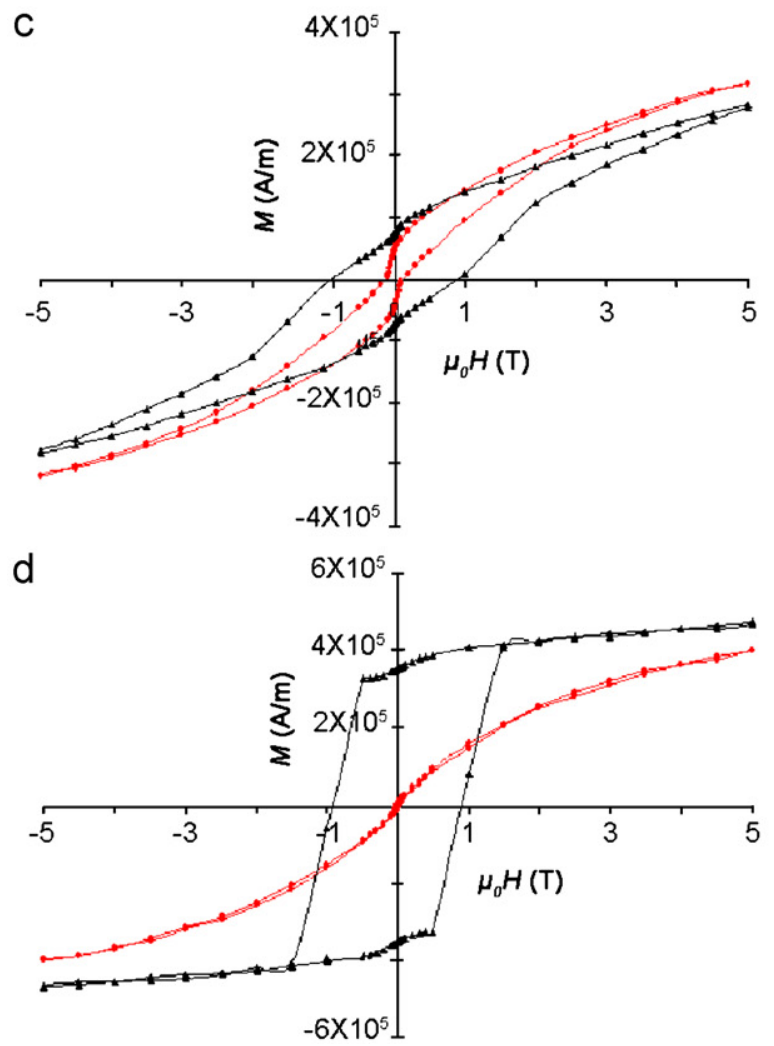

Fig. 5. Hysteresis loops of (Fe $3 \mathrm{~nm} / \mathrm{Dy} 2 \mathrm{~nm}$ ) multilayers deposited at 320 and $570 \mathrm{~K}$, and analyzed at 300 and $100 \mathrm{~K}$. The magnetic field is successively applied in the sample plane (circle dots) and along the normal to the film plane (trianglular dots).

\subsection{1. $T_{\mathrm{S}}=320 \mathrm{~K}$}

Fig. 6 shows the $R^{++}$and $R^{--}$neutron reflectivity curves obtained at $300 \mathrm{~K}$, as well as the fits to the data for the multilayer deposited at $320 \mathrm{~K}$. The applied field was $2 \mathrm{~T}$. The fits were performed with the SimulReflec software [20], considering a stack of $10(\mathrm{Fe} / \mathrm{Dy})$ bilayers with constant individual thicknesses, in agreement with XRR measurements. In order to fit the data, the simplest model was first assumed (regular Fe/Dy superlattice deposited on a silicon substrate). Even though a large number of parameters were let free, no satisfactory fit could be obtained. In order to introduce an extra degree of freedom, the magnetization of the first two bilayers was let to vary freely since HRTEM images (see Fig. 4(b)) suggested that these layers were structurally different from the other bilayers. With this degree of freedom, a quick convergence of the fit was obtained, providing as expected an anti-ferromagnetic coupling between Fe and Dy magnetizations. Other starting points were tested but they did not lead to any acceptable solution. We are confident that the proposed solution is correct since it allows to model multiple sets of data by simply letting the in-plane magnetization vary. The nuclear scattering length density (SLD) and the in-plane magnetization are the adjusted parameters. For the multilayer deposited at $320 \mathrm{~K}$, the nuclear scattering lengths of the Fe and Dy layers are those of the bulk materials, since the mixing between the layers is negligible, as previously observed in CEMS and TAP [19]. The fits to the data give a satisfactory agreement with the experimental data, and therefore enabled the in-plane magnetization of the individual layers to be extracted. Fig. 7 presents the magnetization profile of the sample measured at $300 \mathrm{~K}$. The in-plane magnetization $\left(\mu_{\|}\right)$is expressed in $\left(\mu_{\mathrm{B}} /\right.$ atom). The uncertainty is estimated to be close to $0.2 \mu_{\mathrm{B}} /$ at.

It is important to mention that the total magnetization deduced from the PNR fit $\left(0.6 \times 10^{5} \mathrm{~A} / \mathrm{m}\right)$ is in very good agreement with the experimental value obtained by SQUIDmagnetometry under $2 \mathrm{~T}\left(0.7 \times 10^{5} \mathrm{~A} / \mathrm{m}\right)$. The black arrows represent the direction of the total magnetization $(\boldsymbol{\mu})$ of each layer and $\theta$ is the angle formed by $\boldsymbol{\mu}$ and the normal to the sample plane (n). It must be noted that the magnetization of the multilayer is not fully homogeneous. Indeed, only the first two bilayers deposited on the Si substrate are magnetically ordered, contrary to the rest of the stack. This result is in complete agreement with the HRTEM analysis showing that the first Fe layers deposited on the substrate are mainly crystallized. Consequently, the magnetization measured in the Fe layers deposited first is explained by the presence of bcc-Fe, which has a magnetization of $2.2 \mu_{\mathrm{B}}$ /at. With an in-plane applied field of $2 \mathrm{~T}$, all the magnetic moments are oriented in the film plane and no

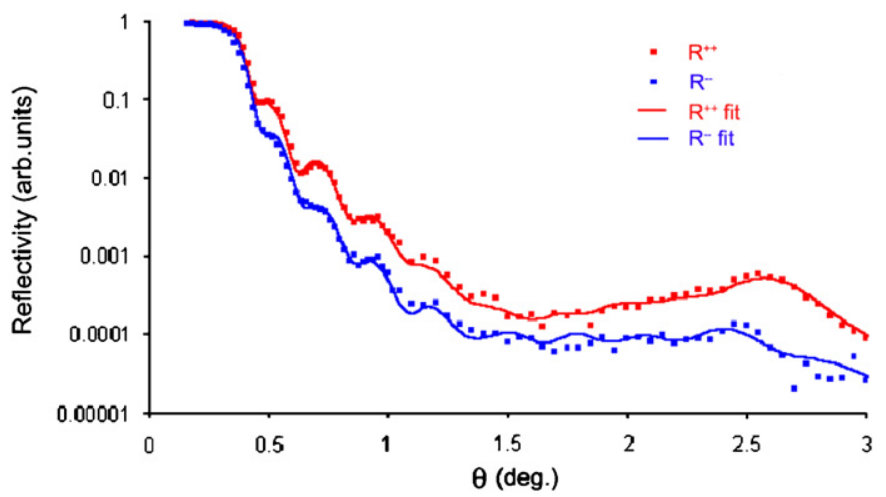

Fig. 6. $R^{++}$and $R^{--}$neutron reflectivity curves of the (Fe $\left.3 \mathrm{~nm} / \mathrm{Dy} 2 \mathrm{~nm}\right)_{10}$ multilayer deposited at $320 \mathrm{~K}$ and measured at $300 \mathrm{~K}$ under an in-plane field of $2 \mathrm{~T}$. 


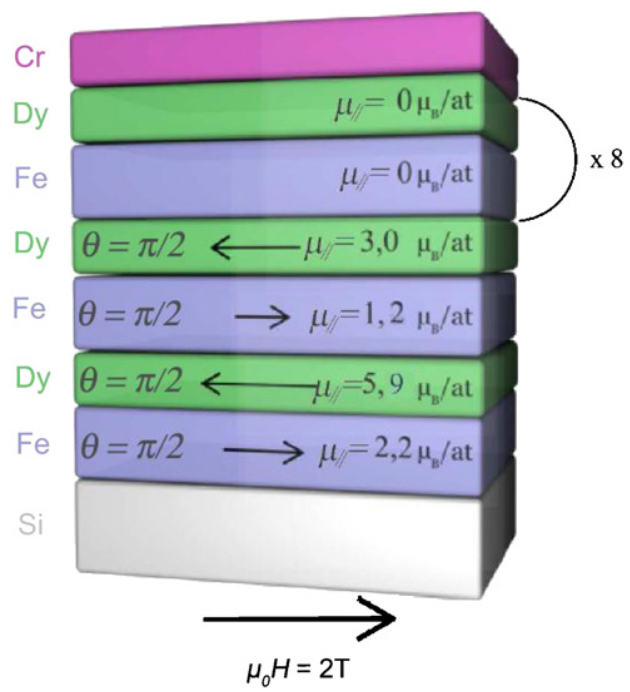

Fig. 7. Magnetization depth profile of the (Fe $3 \mathrm{~nm} / \mathrm{Dy} 2 \mathrm{~nm})_{10}$ multilayer deposited at $320 \mathrm{~K}$ and measured at $300 \mathrm{~K}$.

PMA is observed at room temperature, in agreement with the SQUID measurements (Fig. 5(a)).

Moreover, it is noticeable that the antiparallel configuration of Fe and Dy magnetizations leads to an artificial ferrimagnet. This negative coupling, predicted by the theory of Campbell [24] at the atomic scale, is here clearly evidenced at the scale of nanometric layers as already mentioned by several authors on similar systems [25-27].

The absence of Fe magnetization in the upper layers is explained by a change in the Fe layer structure which becomes amorphous, as observed in Mössbauer spectrometry (Fig. 2). The Curie temperature of pure amorphous $\mathrm{Fe}$ is estimated to be between 200 and $270 \mathrm{~K}[14,28-30]$. Due to the uncertainty on the fits, we cannot evaluate the paramagnetic contribution of the amorphous Fe layers. Thus, their magnetization is considered to be 0 . Concerning the Dy layers, the magnetization is unexpected, since pure amorphous Dy is supposed to be paramagnetic at room temperature $\left(T_{\mathrm{C}}=110 \mathrm{~K}\right)[14,31]$. It should be noted that the first two Dy layers deposited on Si are surrounded by bcc-Fe layers. A polarization effect could therefore be suggested to explain the persistence of magnetization at $300 \mathrm{~K}$. In order to explain qualitatively the magnetization in the first two Dy layers and to validate the PNR fit, a Monte Carlo simulation was performed and will be presented in the next section.

Fig. 8 shows the neutron reflectivity curves of the multilayer deposited at $320 \mathrm{~K}$ and measured at $100 \mathrm{~K}$. The nuclear scattering lengths of $\mathrm{Fe}$ and Dy are the same as at $300 \mathrm{~K}$. Again, the multilayer is magnetically partially inhomogeneous (Fig. 9) due to the presence of $\alpha$-Fe nanocrystals in the first Fe layer (Figs. 2(a), 3(a) and 4(a)). As at $300 \mathrm{~K}$, the first Fe layer exhibits a magnetization of $2.2 \mu_{\mathrm{B}} /$ at. The magnetization of the firstdeposited Dy layer is close to that obtained at $300 \mathrm{~K}$, as will be explained by Monte Carlo simulations. For the upper layers, the in-plane magnetization of the Fe layers $\left(0 \mu_{\mathrm{B}} /\right.$ at) can be interpreted by the existence of PMA or by the formation of a twisted phase, as already seen in (Fe/Gd) multilayers [26,27,32,33]. Indeed, at $100 \mathrm{~K}$, amorphous $\mathrm{Fe}$ is magnetically ordered $[14,28-30]$. However, the small layer thickness and the absence of spin-flip signal are incompatible with the existence of a helicoidal phase. The upper Fe layers are therefore perpendicularly magnetized. Although the SQUID measurements evidence a cone-state, the PNR can only confirm that the $\mathrm{Fe}$ magnetization is on average oriented perpendicular to the film plane. Concerning the upper Dy layers,

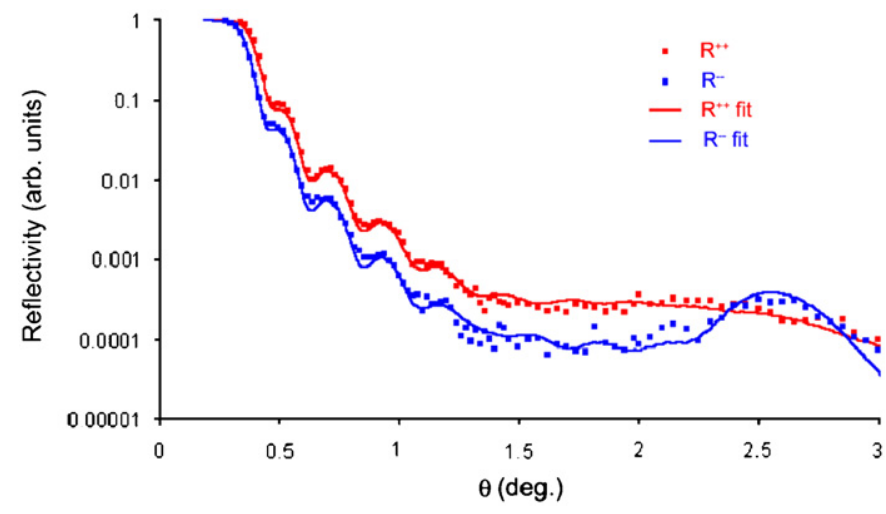

Fig. 8. $R^{++}$and $R^{--}$neutron reflectivity curves of the (Fe $\left.3 \mathrm{~nm} / \mathrm{Dy} 2 \mathrm{~nm}\right)_{10}$ multilayer deposited at $320 \mathrm{~K}$ and measured at $100 \mathrm{~K}$.

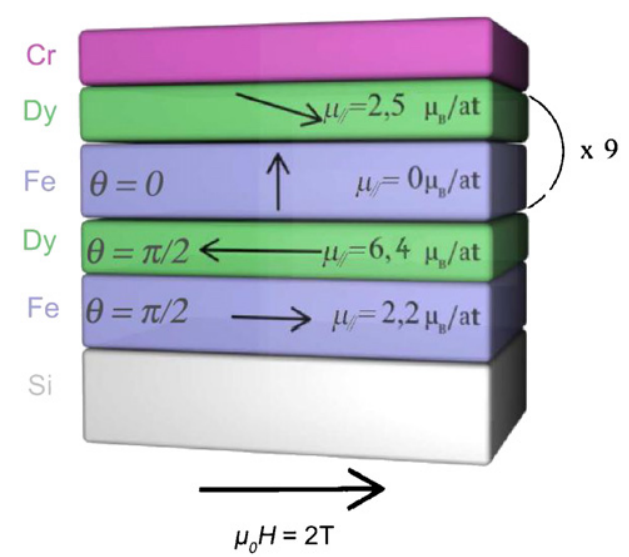

Fig. 9. Magnetization depth profile of the (Fe $3 \mathrm{~nm} / \mathrm{Dy} 2 \mathrm{~nm})_{10}$ multilayer prepared at $320 \mathrm{~K}$ and measured at $100 \mathrm{~K}$.

an in-plane component of $2.5 \mu_{\mathrm{B}}$ /at was measured. However, we did not measure the total magnetization and therefore we cannot give the exact orientation of the Dy layer magnetization. The orientation of the black arrow corresponding to the Dy layer magnetization is arbitrary, but we will see that the Monte Carlo simulations give some information about this orientation.

\subsection{2. $T_{\mathrm{S}}=570 \mathrm{~K}$}

As before, the sample was analyzed at 300 and $100 \mathrm{~K}$ in an inplane field of $2 \mathrm{~T}$. The nuclear scattering length of Fe is the same as in bulk Fe whereas the nuclear SLD attributed to Dy is between the Fe and Dy bulk nuclear scattering lengths. As previously observed by TAP analysis [19], the Dy layers are constituted of an Fe-Dy alloy. In fact, the multilayer prepared at $570 \mathrm{~K}$ consists of a succession of Fe and Fe-Dy alloy layers. Since the PNR curves obtained for $T_{\mathrm{S}}=570 \mathrm{~K}$ look similar to the previous ones, they are not presented. Only the magnetization depth profiles deduced from the PNR fits are shown.

At $300 \mathrm{~K}$, as already observed for $T_{\mathrm{S}}=320 \mathrm{~K}$, the first Fe layer deposited on the Si substrate is magnetically ordered (Fig. 10), which is in agreement with the HRTEM results (Fig. 3), showing some crystallized clusters in the Fe layers, preferentially located near the substrate. The upper Fe layers, structurally disordered, exhibit no in-plane magnetic contribution according to the PNR fit. However, it is clear from the CEMS analysis (Fig. 2(b),(d)) that there is no significant paramagnetic signal. It can therefore be deduced that these Fe layers are ferromagnetic and that their magnetization is perpendicular to the sample plane. For the 


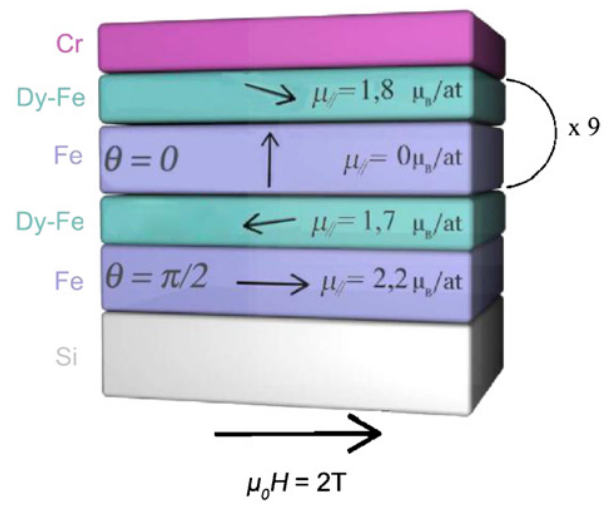

Fig. 10. Magnetization depth profile of the (Fe $3 \mathrm{~nm} / \mathrm{Dy} 2 \mathrm{~nm})_{10}$ multilayer prepared at $570 \mathrm{~K}$ and measured at $300 \mathrm{~K}$.

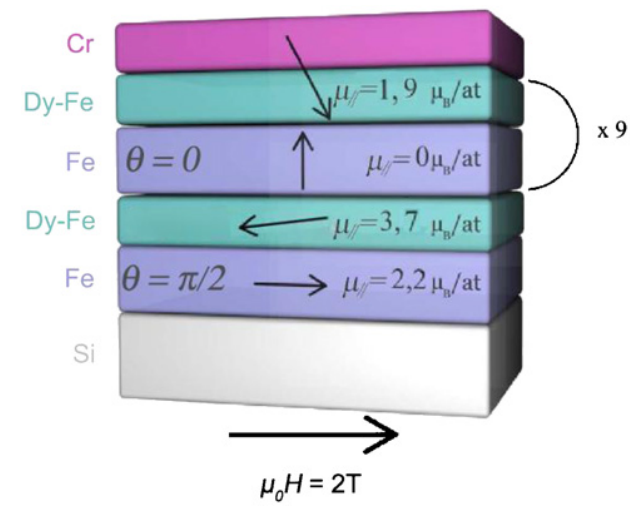

Fig. 11. Magnetization depth profile of the (Fe $3 \mathrm{~nm} / \mathrm{Dy} 2 \mathrm{~nm})_{10}$ multilayer prepared at $570 \mathrm{~K}$ and measured at $100 \mathrm{~K}$.

sample prepared at $320 \mathrm{~K}$, the upper Fe layers were surrounded by non-magnetic Dy layers at $300 \mathrm{~K}$, whereas in this case, the $\mathrm{Fe}$ layers are surrounded by partially magnetically ordered Fe-Dy layers [34]. For $T_{\mathrm{S}}=570 \mathrm{~K}$, the ferromagnetism of the upper $\mathrm{Fe}$ layers at $300 \mathrm{~K}$ is likely to result from a polarization effect due to the ferromagnetic neighboring layers. Concerning the Fe-Dy layers, their magnetization is tilted out of the film plane, since both the SQUID measurements and CEMS spectrum at $300 \mathrm{~K}$ evidenced PMA. However, the tilt angle is unknown and the arrows' orientation is arbitrary. The orientation was qualitatively chosen to satisfy the negative Fe-Dy exchange interactions. Moreover, it can be noticed that the amorphous Fe-Dy alloy layers are magnetically homogeneous, since the in-plane magnetization of the first layer is almost the same as that of the upper layers. As before, the coupling between the first $\mathrm{Fe}$ layer magnetization and the first Dy layer magnetization is negative. Lastly, the sign of the Dy-Fe upper layer in-plane component is opposite to that of the first Dy-Fe layer, because of the Zeeman interaction.

At $100 \mathrm{~K}$ (Fig. 11), the explanation about the first Fe layer is the same as previously. The increase in the first Dy-Fe layer magnetization, compared to that obtained at $300 \mathrm{~K}$, is due to the decrease in the temperature since Dy is magnetically dominant. Concerning the Fe upper layers, the in-plane magnetization of $0 \mu_{\mathrm{B}} /$ at is unambiguously explained by the existence of PMA, as shown on the hysteresis loops (Fig. 5(d)). The phenomenon is equivalent for the Dy-Fe layers which also exhibit a strong PMA. The small in-plane magnetization measured is only due to a strong Zeeman interaction between the Dy magnetic moments and the in-plane applied field. Moreover, the PNR fits lead to a total magnetization $M \approx 2.6 \times 10^{5} \mathrm{~A} / \mathrm{m}$, considering the Fe and Dy bulk densities. This result is in excellent agreement with the magnetization measured by SQUID-magnetometry under $2 \mathrm{~T}$ (Fig. 5(d)) when the magnetic field is applied in the film plane.

In fact, the evolution of the magnetization between $3.7 \mu_{\mathrm{B}} / \mathrm{at}$ for the first Dy-Fe layer and $1.9 \mu_{\mathrm{B}} /$ at for the Dy-Fe upper layers can only be explained by an increase in the PMA through the multilayer. The investigation of (Fe $3 \mathrm{~nm} / \mathrm{Dy} 2 \mathrm{~nm}$ ) multilayers by PNR allowed us therefore to determine a magnetic depth profile through the multilayers. The following information has been deduced:

(1) The multilayers are magnetically not fully homogeneous. In agreement with the HRTEM results, the first layers deposited on the Si substrate are structurally and magnetically different compared to the others.

(2) The exchange coupling between Fe and Dy layers is negative, leading to a ferrimagnetic structure.

\subsection{Monte Carlo simulation}

\subsubsection{Monte Carlo method}

From the HRTEM images, we know that the first-deposited Fe layers are mainly crystallized, contrary to the following Fe layers. This should have a drastic influence on the magnetic parameters of the samples since the exchange interactions are much stronger in crystalline Fe than in amorphous $\mathrm{Fe}$ (the exchange interaction is directly related to the Curie temperature of the material). The amorphous nature of the multilayers was taken into account in the simulation by using a Gaussian distribution of the exchange interaction around the mean value; this distribution results from the distribution of the interatomic distance between nearest neighbors $[28,35]$.

In order to justify the hypothesis used in the PNR fits, in particular the strong in-plane magnetization of the first-deposited layers, we have performed Monte Carlo simulations based on the standard single-flip Metropolis algorithm. The basic system consists of an $(\mathrm{Fe} / \mathrm{Dy} / \mathrm{Fe})$ trilayer in close contact with the substrate, meaning that the Fe layers are mainly composed of $\alpha$-Fe. In this study, we have investigated two different samples (see Fig. 12): the first one (sample A) corresponds to the multilayer deposited at $320 \mathrm{~K}$, characterized by sharp interfaces between Fe and Dy layers, whereas the second one (sample B) is representative of the multilayer prepared at $570 \mathrm{~K}$, which shows a large diffusion of Fe atoms in the Dy layer. These simulations aim at studying the influence of the concentration profile on the magnetization induced in the Dy layer by the surrounding Fe layers. The layers' thicknesses have been chosen close to the

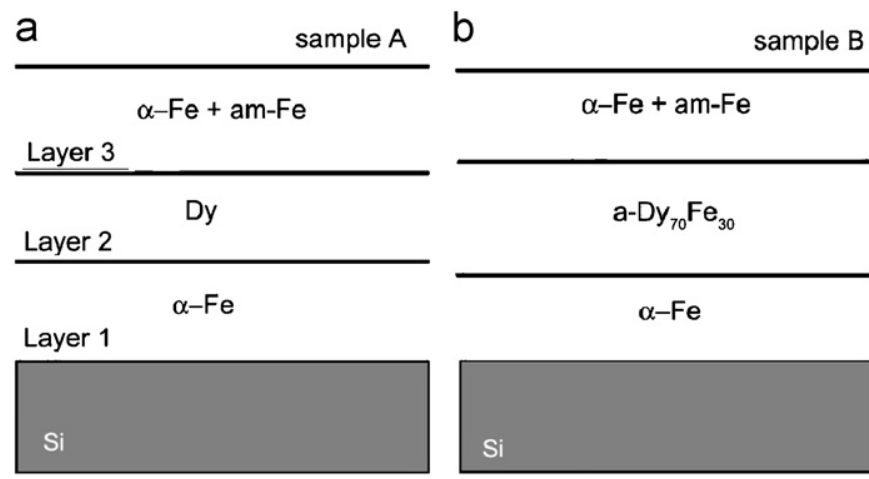

Fig. 12. Schematic representation of the two samples named A and B investigated by Monte Carlo simulations. They are, respectively, associated with the real multilayers prepared at $320 \mathrm{~K}$ (a) and $570 \mathrm{~K}$ (b). 'am' stands for amorphous. 
experimental ones ( $3 \mathrm{~nm}$ for Fe and $2 \mathrm{~nm}$ for Dy) and lead to an overall concentration of Fe atoms of $80 \%$ in each case. The model used in these simulations consists of classical Heisenberg spins $\mathbf{S}_{i}$ of two different species (Fe and Dy), located at the nodes of a facecentered cubic lattice of 450 atoms per atomic plane, with periodic boundary conditions in the plane and free boundary conditions in the deposition direction (arbitrarily chosen as the $z$ axis). In a first attempt to get a qualitative description of the magnetic behavior of our real samples, we have neglected the anisotropy energy. According to the possible values of the Dy local anisotropy constant $[2,12]$, the exchange energy is indeed much larger than the anisotropy energy. The Hamiltonian $\mathscr{H}$ is therefore given by

$\mathscr{H}=-\sum_{\langle i, j\rangle} J_{i j} \mathbf{S}_{i} \cdot \mathbf{S}_{j}-\mu_{0} \mathbf{H} \cdot \sum_{i} \mathbf{m}_{i}$,

where $J_{i j}$ is the exchange interaction between nearest neighbors, $\mathbf{H}$ is the magnetic field applied in the sample plane $\left(\mu_{0} H=2 \mathrm{~T}\right)$ and $\mathbf{m}_{i}$ is the magnetic moment of the site $i\left(m_{\mathrm{Fe}}=g_{\mathrm{Fe}} \mu_{\mathrm{B}} S_{\mathrm{Fe}}\right.$ and $\left.m_{\text {Dy }}=g_{\text {Dy }} \mu_{\mathrm{B}} J_{\text {Dy }}=\left(g_{\text {Dy }} /\left(g_{\text {Dy }}-1\right)\right) \mu_{\mathrm{B}} S_{\text {Dy }}\right)$. Some magnetic parameters have the standard values of the free atoms $\left(g_{\mathrm{Fe}}=2, g_{\mathrm{Dy}}=\right.$ $\frac{4}{3}$ and $S_{\mathrm{Dy}}=5 / 2$ ) whereas the values of the exchange interactions $J_{i j}$ and of the Fe spin $S_{\mathrm{Fe}}$ are taken from the experimental work of Heiman on Dy-Fe amorphous thin films [30] and adjusted in order to get the pure amorphous Fe and Dy Curie temperatures:

$J_{\mathrm{Fe}-\mathrm{Fe}}\left(X_{\mathrm{Fe}}\right)=20.5+120\left(1-X_{\mathrm{Fe}}\right) \quad\left(\right.$ in $\left.10^{-23} \mathrm{~J}\right)$,

$J_{\mathrm{Fe}-\mathrm{Dy}}\left(X_{\mathrm{Fe}}\right)=2.25-53\left(1-X_{\mathrm{Fe}}\right) \quad\left(\right.$ in $\left.10^{-23} \mathrm{~J}\right)$,

$J_{\text {Dy-Dy }}=1.6$ (in $\left.10^{-23} \mathrm{~J}\right)$,

and

$S_{\mathrm{Fe}}\left(X_{\mathrm{Fe}}\right)=1.1-1.125\left(1-X_{\mathrm{Fe}}\right)$,

where $X_{\mathrm{Fe}}$ is the Fe concentration in the Dy-Fe alloy. If Fe is crystallized in the $\alpha$ phase, the exchange integrals are

$J_{\mathrm{Fe}^{\alpha}-\mathrm{Fe}^{\alpha}}=82, \quad J_{\mathrm{Fe}^{\alpha}-\mathrm{Dy}}=-16 \quad$ (in $10^{-23} \mathrm{~J}$ )

It is important to note that the exchange interaction between Fe and Dy spins is strongly negative, leading to ferrimagnetic structures in both alloys and multilayers. The numerical procedure is the importance-sampling Monte Carlo method [36-38] associated with the standard Metropolis algorithm [39]. Starting from a random configuration of the direction of each spin in the paramagnetic phase, a single spin is randomly chosen and a new orientation is proposed. If the energy variation $\Delta E$ corresponding to this transition is negative, the flip is automatically accepted, whereas in the opposite case, the transition is accepted only with a probability that is proportional to the Boltzmann factor $\exp \left(-\Delta E / k_{\mathrm{B}} T\right)$. This condition of acceptance of the transition allows the system to escape from a local minimum in the free energy landscape when the Hamiltonian does not display too much frustration, which is the case here. One Monte Carlo step (MCS) consists in visiting all the spins of the system once. During the simulation, it is necessary to reach equilibrium at each temperature, which is why we performed 5000 MCS for the thermalization, and afterwards the thermodynamic quantities were measured over 10,000 MCS. This procedure allows reliable values of the physical properties under interest to be obtained, since they do not depend on the randomly generated initial state.

\subsubsection{Monte Carlo results}

Fig. 13(a) shows the thermal variation of the magnetization of sample A and of the three individual layers, as well as the projections along the direction of the applied magnetic field. The magnetization values are divided by the saturated magnetization (total or layer by layer); consequently, the magnetization scale

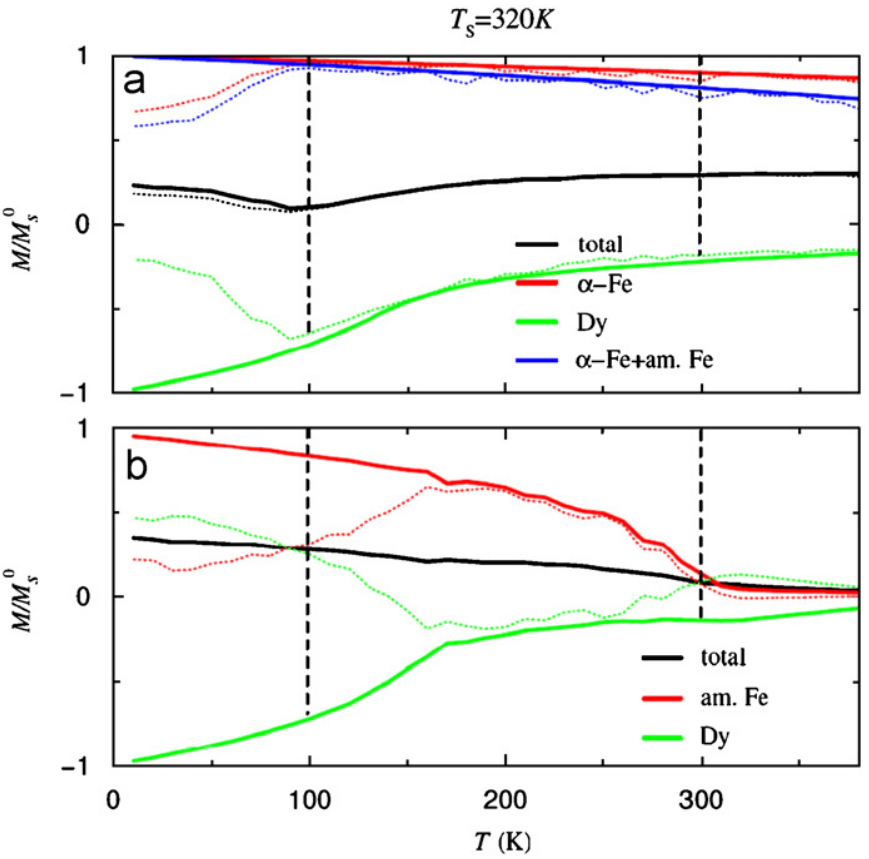

Fig. 13. Variation of the reduced magnetization of sample $A$ and of the individual layers as a function of the temperature (solid lines) (a). (b) Variation of a Dy layer surrounded by two amorphous Fe layers. The dotted curves correspond to the projection on the applied magnetic field direction.

varies from -1 to +1 because of the negative exchange interaction between $\mathrm{Fe}$ and Dy $\left(\mathrm{J}_{\mathrm{Fe}-\mathrm{Dy}}<0\right)$.

At $T=300 \mathrm{~K}$, the Fe layers are strongly magnetized, slightly more for the crystalline phase (layer 1) than for the layer containing both amorphous and crystalline Fe (layer 3). This is due to the value of the exchange interaction being larger in $\alpha$-Fe than in amorphous Fe. Although the critical temperature of pure amorphous Dy is around $110 \mathrm{~K}[14,31]$, we note that the proximity effect with the Fe layers leads to a remanent magnetization of $0.2 M_{\mathrm{S}} \sim 2 \mu_{\mathrm{B}} /$ at. These results confirm qualitatively the PNR fit that shows a non-zero magnetization of the first two Dy layers at room temperature. When the temperature decreases, the Dy layer orders progressively, leading to a decreasing value of the net magnetization.

At $T=100 \mathrm{~K}$, just above the magnetic compensation temperature, the Dy layer is quite strongly magnetized along the applied field $\left(M_{\mathrm{H}} \sim 0.65 M_{\mathrm{S}}\right)$ which gives a magnetic moment per atom of $6.5 \mu_{\mathrm{B}}$, as observed in PNR measurements. We can thus summarize the magnetic ordering of sample A as shown in Fig. 14.

From the simulation, we can extrapolate the magnetic configuration at low temperature $(T=10 \mathrm{~K})$. Below $100 \mathrm{~K}$, we observe the self-ordering of the Dy layer along an indifferent direction due to the competition between the Zeeman energy with the $\mathrm{Fe}$ and Dy magnetic moments and the interfacial exchange energy.

This orientation of the magnetization at low temperature in a direction which is not necessarily that of the magnetic field can be explained by the calculation of the energy taking into account the mean exchange interactions between nearest-neighbor atoms. Let us assume a ferromagnetic order in each layer and define an angle $\alpha$ between the directions of the Dy moments and the applied magnetic field and an angle $\beta$ between the Dy and Fe moments (Fig. 15(a)); we then determine the energy of the trilayer as a function of the parameters $\alpha$ and $\beta$. The result is shown in Fig. 15(b) where we can see that the minimum, as expected, corresponds to $\alpha=0$ and $\beta=\pi$, meaning that a ferrimagnetic 
order exists along the direction of the applied magnetic field. But we can observe that this minimum is rather flat along the $\alpha$-axis and that a variation of the parameter $\alpha$ from 0 to 1 rad leads to a small variation of the energy

$E(\alpha=1, \beta=0)-E_{\min } \simeq 13 \mathrm{~K} /$ at.

This result indicates that at low temperature, the direction of the magnetization cannot be known precisely since even small thermal fluctuations are strong enough to induce a perturbation in the magnetization axis.
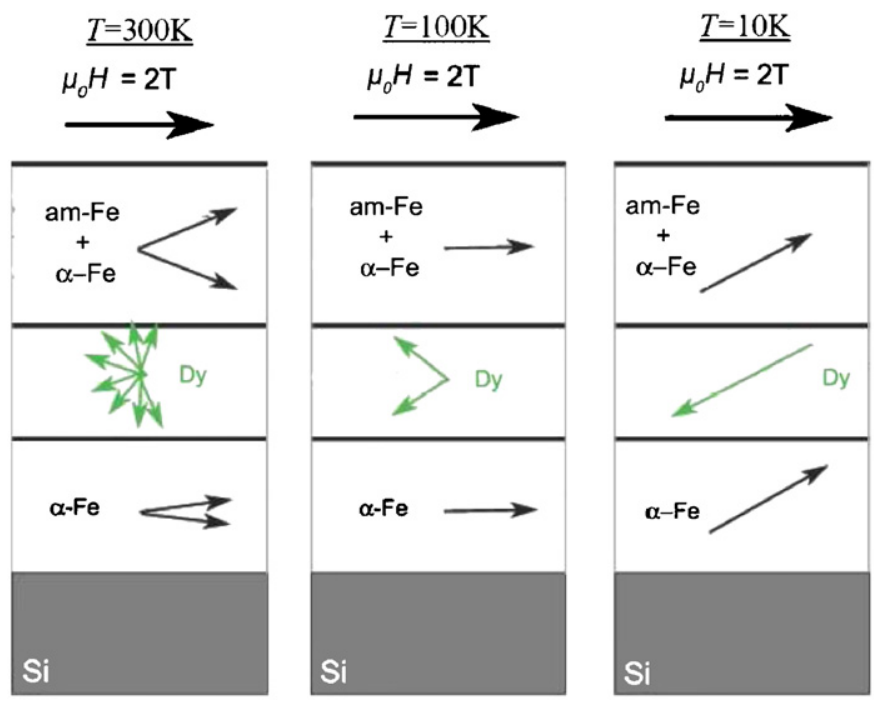

Fig. 14. Schematic representation of the magnetic ordering of sample A when the temperature decreases.
In order to estimate the orientation of the Dy magnetization in the upper layers, simulations were performed on a trilayer made of a Dy layer sandwiched between two amorphous Fe layers (Fig. 13(b)). We can note that the modulus of the Dy magnetization at $T=100 \mathrm{~K}$ is close to the previous one $\left(M \approx 0.64 M_{\mathrm{S}}^{0}\right.$ to be compared to $0.65 M_{\mathrm{S}}^{0}$ ). This result shows that at $T=100 \mathrm{~K}$ the modulus of the Dy magnetization is almost independent of the structure of the surrounding Fe layers. On the contrary, its component along the direction of the applied field is very sensitive to the Fe layer structure since the absolute value is much lower in the case of am-Fe $\left(M_{\mathrm{H}} \sim 0.3 M_{\mathrm{S}}^{0}\right)$ than in the case of $\alpha$-Fe $\left(M_{\mathrm{H}} \sim 0.65 M_{\mathrm{S}}^{0}\right)$. Moreover, the sign of the Dy magnetization projection of Fig. 13(b) is opposite to that of Fig. 13(a). We may interpret this result as an effect of the competition between the Zeeman interaction and the exchange interactions. This is in total agreement with the experimental magnetization profile presented in Fig. 9 and is attributed to the lower degree of frustration in the upper Dy layers due to the perpendicular orientation of the $\mathrm{Fe}$ layers' magnetization.

For the other multilayer, deposited at $570 \mathrm{~K}$, the main structural difference comes from the diffusion of $\mathrm{Fe}$ atoms in the Dy layer. The numerical results obtained with sample $B$ are shown in Fig. 16. The data concerning the Fe layers (first and third layers) are almost the same as in the previous case. For the second layer, consisting in an amorphous $\mathrm{Dy}_{0.7} \mathrm{Fe}_{0.3}$ alloy, it is to be noted that the reduced magnetization is obtained here by dividing by the ferrimagnetic magnetization and not the saturated magnetization with all Dy and Fe magnetic moments aligned parallel to each other.

Indeed, due to the strong anti-ferromagnetic coupling between Fe and Dy magnetic moments, the stable magnetic structure at $0 \mathrm{~K}$ is obtained with all the Dy moments pointing antiparallel to the Fe moments. Thus, the mean magnetic moment per atom in the second layer is $0.7 \times 10-0.3 \times 2.2 \sim 6.3 \mu_{\mathrm{B}}$ instead of $10 \mu_{\mathrm{B}}$ as it

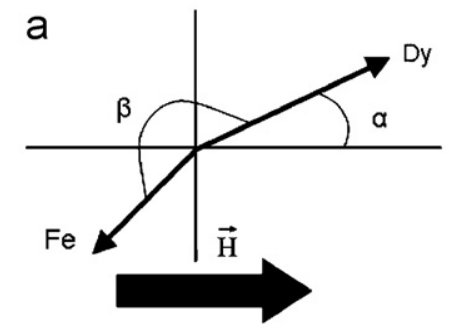

b

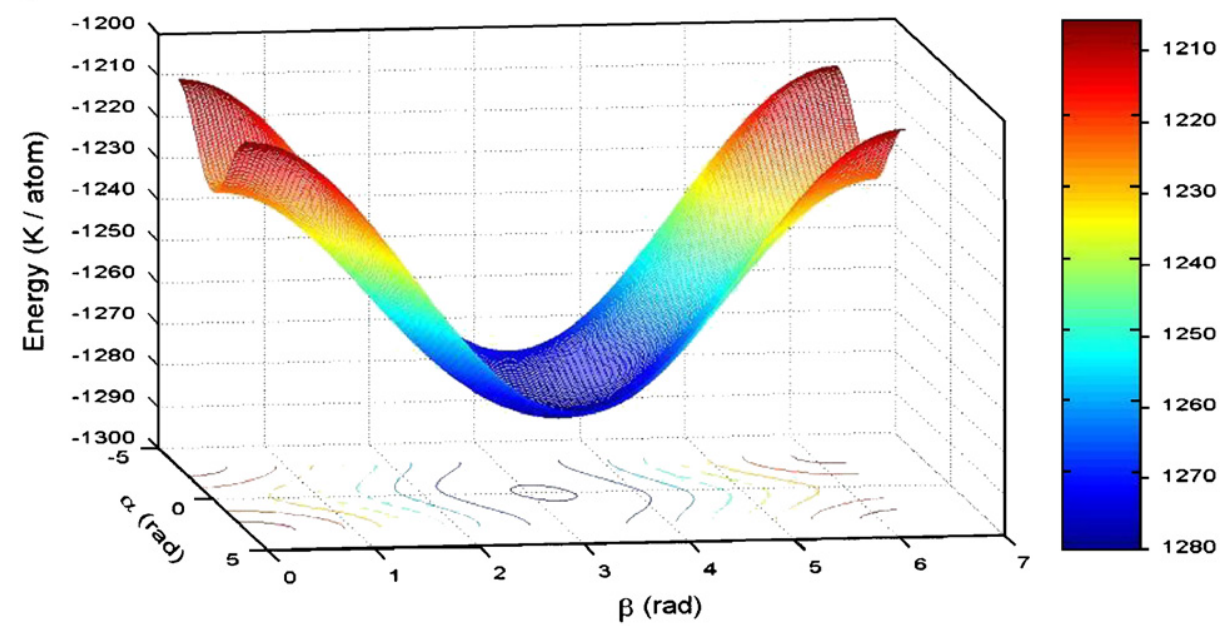

Fig. 15. (a) Definition of the angles $\alpha$ and $\beta$ used to calculate the energy of the trilayer. (b) Variation of the energy of the fundamental state as a function of the angles $\alpha$ and 


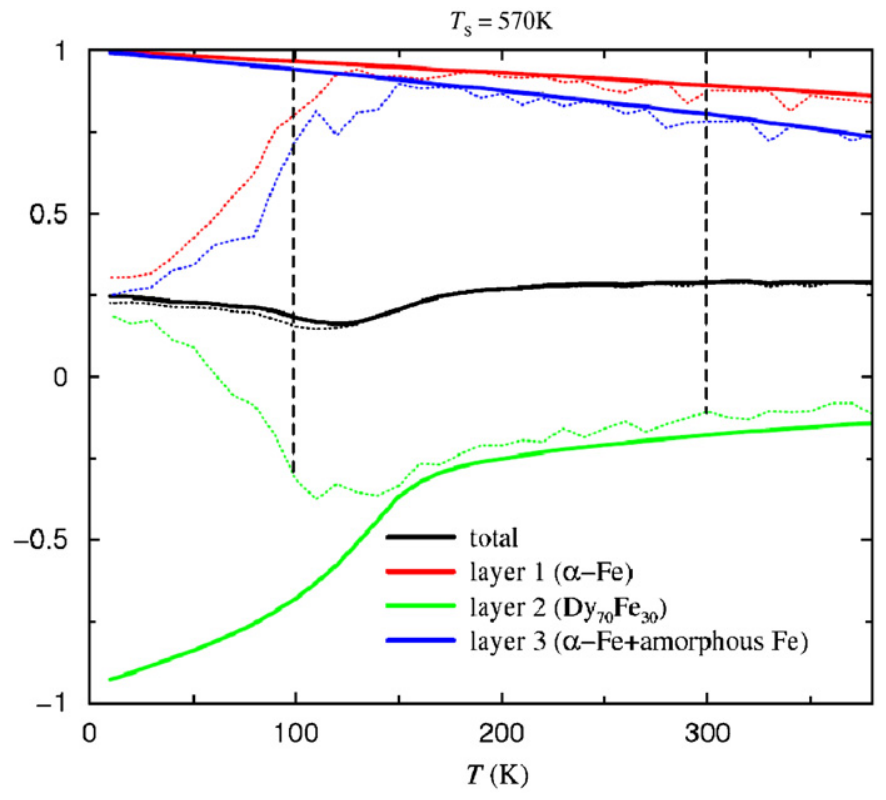

Fig. 16. Variation of the reduced magnetization of sample $B$ and of the three individual layers as a function of the temperature (solid lines). Projection of the magnetization on the applied field direction (dotted lines).

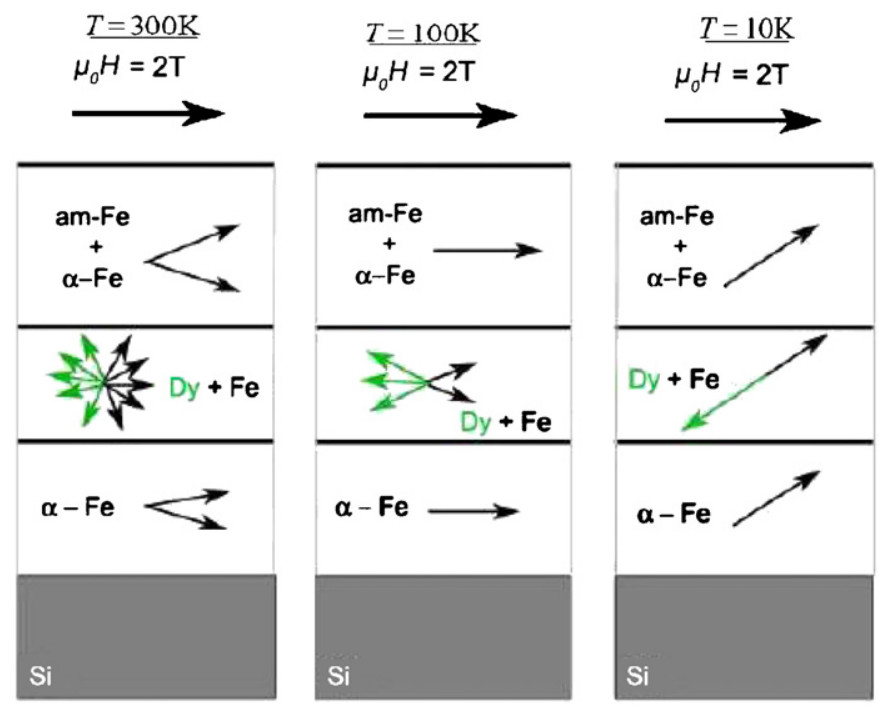

Fig. 17. Schematic representation of the magnetic ordering of sample B when the temperature decreases.

was for sample A. We therefore obtain at $300 \mathrm{~K}$ a magnetic moment per atom along the applied field direction of around $0.12 \times 6.3 \sim 0.8 \mu_{\mathrm{B}}$, which is 2.5 times smaller than the value calculated for sample A. We can also observe that the compensation point is located at higher temperature than for the previous sample because of the chemical mixing between the two elements.

At $100 \mathrm{~K}$, even if the Dy-Fe layer is still strongly magnetized, the magnetization component along the applied field is reduced to about $2.2 \mu_{\mathrm{B}}$. In this case, the magnetic ordering when the temperature decreases is depicted in Fig. 17.

Again, at low temperature, the direction of the magnetic moments is indifferent and depends on the competition in the Zeeman interaction between the Fe and Dy sublattices. It is important to mention that we are not able, with this simple model, to evidence the existence of PMA, since we did not include any magnetic anisotropy in the Hamiltonian.

\section{Conclusion}

Polarized neutron reflectometry allowed us to determine magnetic depth profiles in (Fe/Dy) multilayers deposited in different evaporation conditions. The ferrimagnetic order of the samples was evidenced, as well as the perpendicular magnetic anisotropy, that was found to persist at room temperature for the multilayer deposited at $570 \mathrm{~K}$. The detailed magnetic depth profiling has pinpointed the key role of the crystalline structure of Fe as well as the role of Dy-Fe alloys in the magnetic order. The role of the proximity effects between the layers on the magnetic order was demonstrated using Monte Carlo simulations.

\section{Acknowledgments}

The authors wish to thank Dr. Etienne Snoeck for the high quality HRTEM images. Moreover, this work was supported by the computer-time Grant \#2004002 of the Centre de Ressources Informatiques de Haute Normandie (CRIHAN).

\section{References}

[1] Z.S. Shan, D.J. Sellmyer, S.S. Jaswal, Y.J. Wang, J.X. Shen, Phys. Rev. Lett. 63 (1989) 449.

[2] Z.S. Shan, D.J. Sellmyer, Phys. Rev. B 42 (1990) 10433.

[3] Z.S. Shan, D.J. Sellmyer, S.S. Jaswal, Y.J. Wang, J.X. Shen, Phys. Rev. B 42 (1990) 10446.

[4] K. Mibu, N. Hosoito, T. Shinjo, J. Magn. Magn. Mater. 126 (1993) 343.

[5] B. Scholz, R.A. Brand, W. Keune, Phys. Rev. B 50 (1994) 2537.

[6] W.S. Kim, W. Kleemann, J. Tappert, W. Keune, J. Appl. Phys. 84 (1998) 4384

[7] N. Sato, K. Habu, J. Appl. Phys. 61 (1987) 4287.

[8] M. Piecuch, L.T. Baczewski, J. Durand, G. Marchal, P. Delcroix, H. Nabli, J. Physique C8 (1988) 1755.

[9] S. Honda, M. Nawate, I. Sakamoto, J. Appl. Phys. 79 (1996) 365.

[10] J. Tejada, F. Badia, B. Martinez, J.M. Ruiz, J. Magn. Magn. Mater. 101 (1991) 181.

[11] K. Attenkofer, S. Stähler, M. Knülle, P. Fisher, G. Schütz, G. Wiesinger, B. Scholz, J. Appl. Phys. 73 (1993) 6910.

[12] Y.J. Wang, C.P. Luo, W. Kleemann, B. Scholz, R.A. Brand, W. Keune, J. Appl. Phys 73 (1993) 6907.

[13] M. Sajieddine, Ph. Bauer, C. Dufour, K. Cherifi, G. Marchal, Ph. Mangin, J. Magn Magn. Mater. 113 (1992) 243.

[14] J. Tappert, W. Keune, R.A. Brand, P. Vulliet, J.-P. Sanchez, T. Shinjo, J. Appl. Phys 80 (1996) 4503.

[15] W.-S. Kim, W. Andrä, W. Kleemann, Phys. Rev. B 58 (1998) 6346

[16] T. Ruckert, W. Keune, W. Sturhan, E.E. Alp, J. Magn. Magn. Mater. 240 (2002) 562.

[17] F. Yang, F. Pan, Physica B 315 (2002) 227.

[18] T. Shinjo, K. Yoden, N. Hosoito, J.P. Sanchez, J.M. Friedt, J. Phys. Soc. Jap. 58 (1989) 4255.

[19] A. Tamion, E. Cadel, C. Bordel, D. Blavette, Scripta Mater. 54 (2006) 671.

[20] 〈http://www-llb.cea.fr/prism/programs/simulreflec/simulreflec.html〉.

[21] T. Ruckert, J. Tappert, R. Brand, W. Keune, J. Magn. Magn. Mater. 165 (1997) 411.

[22] R.C. Taylor, T.R. McGuire, J.M.D. Coey, A. Gangulee, J. Appl. Phys. 49 (1978) 2885.

[23] J. Tappert, S. Neumann, R.A. Brand, W. Keune, F. Klose, H. Maletta, Europhys Lett. 46 (1999) 238.

[24] I.A. Campbell, J. Phys.: Metal. Phys. 2 (1972) L47.

[25] M. Taborelli, R. Allenspach, G. Boffa, M. Landolt, Phys. Rev. Lett. 56 (1986) 2869.

[26] C. Dufour, K. Cherifi, G. Marchal, Ph. Mangin, M. Hennion, Phys. Rev. B 47 (1993) 14572.

[27] W. Hahn, M. Loewenhaupt, Y.Y. Huang, G.P. Felcher, S.S.P. Parkin, Phys. Rev. B 52 (1995) 16041.

[28] N. Heiman, N. Kazama, Phys. Rev. B 19 (1979) 1623.

[29] D.H. Ryan, J.M.D. Coey, E. Batalla, Z. Altounian, J.O. Ström-Olsen, Phys. Rev. B 35 (1987) 8630

[30] N. Heiman, K. Lee, R.I. Potter, S. Kirkpatrick, J. Appl. Phys. 47 (1976) 2634.

[31] M.K. Wilkinson, W.C. Koehler, E.O. Wollan, J.W. Cable, J. Appl. Phys. 32 (1961) S48.

[32] R.E. Camley, D.R. Tilley, Phys. Rev. B 37 (1988) 3413.

[33] K. Cherifi, C. Dufour, Ph. Bauer, G. Marchal, Ph. Mangin, Phys. Rev. B 44 (1991) 7733. 
[34] P. Hansen, S. Klahn, C. Clausen, G. Much, K. Witter, J. Appl. Phys. 69 (1991) 3194.

[35] J.M.D. Coey, J. Appl. Phys. 49 (1978) 1646.

[36] D.W. Heermann, Computer Simulation Methods in Theoretical Physics, second ed., Springer, Berlin, 1990.
[37] K. Binder, D.W. Heermann, Monte Carlo Simulation in Statistical Physics, second ed., Springer, Berlin, 1990.

[38] P.K. Mac Keown, Stochastic Simulation in Physics, Springer, Berlin, 1997.

[39] N. Metropolis, A.E. Rosenbluth, M.N. Rosenbluth, A.H. Teller, E. Teller, J. Chem. Phys. 21 (1953) 1087. 